\title{
Element-Specific X-Ray Phase Tomography of 3D Structures at the Nanoscale
}

\author{
Claire Donnelly, ${ }^{1,2}$ Manuel Guizar-Sicairos, ${ }^{2,}$ Valerio Scagnoli, ${ }^{1,2}$ Mirko Holler, ${ }^{2}$ Thomas Huthwelker, ${ }^{2}$ Andreas Menzel, ${ }^{2}$ \\ Ismo Vartiainen, ${ }^{2}$ Elisabeth Müller, ${ }^{2}$ Eugenie Kirk, ${ }^{1,2}$ Sebastian Gliga, ${ }^{1,2}$ Jörg Raabe, ${ }^{2}$ and Laura J. Heyderman ${ }^{1,2, \dagger}$ \\ ${ }^{1}$ Laboratory for Mesoscopic Systems, Department of Materials, ETH Zurich, 8093 Zurich, Switzerland \\ ${ }^{2}$ Paul Scherrer Institute, 5232 Villigen PSI, Switzerland \\ (Received 23 October 2014; published 16 March 2015)
}

\begin{abstract}
Recent advances in fabrication techniques to create mesoscopic 3D structures have led to significant developments in a variety of fields including biology, photonics, and magnetism. Further progress in these areas benefits from their full quantitative and structural characterization. We present resonant ptychographic tomography, combining quantitative hard x-ray phase imaging and resonant elastic scattering to achieve $a b$ initio element-specific 3D characterization of a cobalt-coated artificial buckyball polymer scaffold at the nanoscale. By performing ptychographic x-ray tomography at and far from the Co $K$ edge, we are able to locate and quantify the Co layer in our sample to a 3D spatial resolution of $25 \mathrm{~nm}$. With a quantitative determination of the electron density we can determine that the Co layer is oxidized, which is confirmed with microfluorescence experiments.
\end{abstract}

DOI: 10.1103/PhysRevLett.114.115501

With recent advances in technology opening up the possibilities of true three-dimensional (3D) nano- and microfabrication [1], new opportunities for applications have emerged within a large number of fields [1,2]. Extending metamaterials into the third dimension allows increased control over photonic properties, such as the introduction of structural chirality resulting in broadband circular polarizing effects [3]. With a quasi-3D system, the first complete photonic band gap at near-IR frequencies was achieved [4], making the 3D regime an obvious route for further advances in photonics. Three-dimensional structures are also of great interest in magnetism enabling, for example, high-density data storage architectures [5] and the control of magnetic properties [6]. Furthermore, 3D advanced materials have been employed in many other fields, with the potential for the manipulation of mechanical properties [7] and for medical applications [2] such as drug delivery with fabricated 3D magnetic microbots [8]. The quantitative characterization of these designed materials, as well as other complex materials including catalysts or composites [9], is essential to further improve their performance.

Here we present the full structural and elemental characterization of a polymer-metal artificial buckyball at $25 \mathrm{~nm}$ spatial resolution by resonant $\mathrm{x}$-ray ptychographic tomography. While ptychography measurements across absorption edges have been demonstrated in 2D [10-12], to our knowledge this is both the first demonstration of 3D resonant ptychography and the first time that phase contrast off and on resonance has been combined to determine the atomic density of a specific element. In contrast to nonresonant phase tomography, which relies on having different electron densities for different phases [13], resonant ptychographic tomography is element specific, allowing not only for an element to be located but also for further
PACS numbers: 81.07.-b, 42.30.Rx, 68.37.Yz, 81.70.Tx

information on its chemical state to be determined, so providing a unique tool for the characterization of $3 \mathrm{D}$ materials at the nanoscale.

$\mathrm{X}$-ray ptychography is a scanning variant of coherent diffractive imaging $[14,15]$ and provides access to the full complex transmission function, $T$, of a specimen with both phase, $\phi$, and amplitude, $A$, contrast:

$$
T(x, y)=A e^{j \phi}=\exp \left(-\frac{2 \pi}{\lambda} \int[\beta(\mathbf{r})+j \delta(\mathbf{r})] d z\right)
$$

where $\mathbf{r}=(x, y, z)$ is the $3 \mathrm{D}$ set of Cartesian coordinates with $z$ the direction of $\mathrm{x}$-ray propagation and $n=1-\delta-j \beta$ the complex refractive index. For $x$-ray ptychography, the sample is illuminated with a coherent beam, and far-field diffraction patterns are recorded for many overlapping areas. The resulting data redundancy allows for the reconstruction of both the illumination and the specimen [15-17]. This ability to quantitatively measure the phase, which provides superior contrast compared to absorption at hard x-ray energies, allows high-contrast imaging of largescale $\mu \mathrm{m}$-sized structures. The spatial resolution is limited by the scattering angle of the measurable diffraction pattern and the accuracy and stability of the positioning of the sample [18], currently reaching $5 \mathrm{~nm}$ in 2D [19] and $16 \mathrm{~nm}$ in 3D [20]. Here we demonstrate that combining phase tomography with the probing of element-specific resonances enables the determination of both the atomic and electron density, providing information on both the location and electronic state of atoms of a specific element with high spatial resolution.

An artificial buckyball polymer scaffold was fabricated by two-photon absorption lithography using a Nanoscribe 3D direct laser write system with the negative-tone Nanoscribe IP-Dip resist. To ensure a clear $360^{\circ}$ view of 
the sample for tomographic imaging, the buckyball was fabricated on top of a high aspect ratio silicon pillar of height $36 \mu \mathrm{m}$ and diameter $10 \mu \mathrm{m}$, which was produced using a combination of electron beam lithography and reactive-ion etching performed with the Bosch process [21]. The buckyball was $6 \mu \mathrm{m}$ in diameter with a width of the connecting bars of around $240 \mathrm{~nm}$ and was coated with $30 \mathrm{~nm}$ of Co capped with $6 \mathrm{~nm}$ of gold using an ultrahigh vacuum sputtering system. As a final step, the 3D nanostructure was transferred and attached to the sample mount using a focused ion beam and micromanipulator. Scanning electron micrographs of the sample on the tomography pin are given in Fig. 1.

For ptychographic tomography [22], 2D projections are measured at a number of sample orientations with respect to the incident $\mathrm{x}$-ray beam. Here, these were performed at a photon energy of $6.20 \mathrm{keV}$ and then at $7.71 \mathrm{keV}$, where the latter energy corresponds to the $K$ absorption edge (the $1 s-4 p$ transition) of cobalt. The experiments were performed at the cSAXS beam line at the Swiss Light Source, Paul Scherrer Institute, Switzerland, using an interferometrically controlled high-resolution 3D scanning setup $[20,23]$, which allows for nanometer precision positioning combined with full sample rotation over $360^{\circ}$. The illumination on the sample was defined by a combination of a $40 \mu \mathrm{m}$ central stop, a $30 \mu \mathrm{m}$ order-sorting aperture, and gold Fresnel zone plates with $1.2 \mu$ m gold thickness, $60 \mathrm{~nm}$ outermost zone width, and $170 \mu \mathrm{m}$ and $120 \mu \mathrm{m}$ diameters for photon energies of $6.20 \mathrm{keV}$ and $7.71 \mathrm{keV}$, respectively, giving similar focal distances that accommodate for the mechanical constraints of the setup.

Ptychography scans with a field of view of $10 \mu \mathrm{m} \times$ $10 \mu \mathrm{m}$ were performed on a circular grid with a spacing of $0.5 \mu \mathrm{m}$ between the circular shells and 5 points in the center shell. A diffraction pattern was measured at each of 323 scanning points with a 0.2 second exposure time using a Pilatus detector [24,25] at a distance of $7.38 \mathrm{~m}$ from the sample. Including sample and detector positioning [20], each projection took approximately $3 \mathrm{~min}$, with each tomogram composed of 160 projections equally distributed over $180^{\circ}$, administering an estimated total dose of $116 \mathrm{MGy}$ and $19 \mathrm{MGy}$ to the cobalt and resist, respectively. Ptychographic reconstructions were performed with the data from $400 \times 400$ pixels of the detector, resulting in

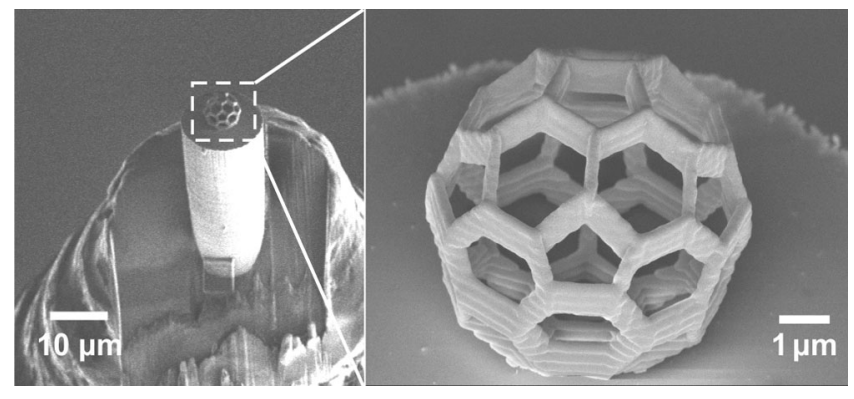

FIG. 1. Scanning electron micrographs of the Co-coated artificial buckyball mounted on the tomography pin. pixel sizes of $21.48 \mathrm{~nm}$ and $17.27 \mathrm{~nm}$ for the $6.20 \mathrm{keV}$ and $7.71 \mathrm{keV}$ reconstructions, respectively. For the reconstruction we applied 400 iterations of the difference map [15], followed by 200 iterations of a nonlinear optimization routine for maximum likelihood estimation [26].

The reconstructed amplitude and phase of one of the 2D projections taken at an energy of $6.20 \mathrm{keV}$ can be seen in Figs. 2(a) and 2(b), respectively. The cobalt layer around the buckyball bars lying parallel to the x-ray beam has relatively high contrast in the amplitude projection as indicated by the arrows in Fig. 2(a). It is only possible, however, to see the full structure in the reconstruction of the phase, which has a much higher signal to noise ratio, as seen in Fig. 2(b). Here the bars perpendicular to the x-ray beam can now be seen clearly.

To quantify the atomic density of a specific element, we first need to consider the complex refractive index, $n$, which, for the case of small-angle scattering resulting from the interaction of $\mathrm{x}$ rays with the electrons of the sample, can be expressed in terms of the atomic scattering factor $f_{0}=f_{1}+j f_{2}[27]$ :

$$
n=1-\delta-j \beta=1-\frac{r_{\mathrm{e}}}{2 \pi} \lambda^{2} \sum_{k} n_{\mathrm{at}}^{k}\left(f_{1}^{k}+j f_{2}^{k}\right)
$$
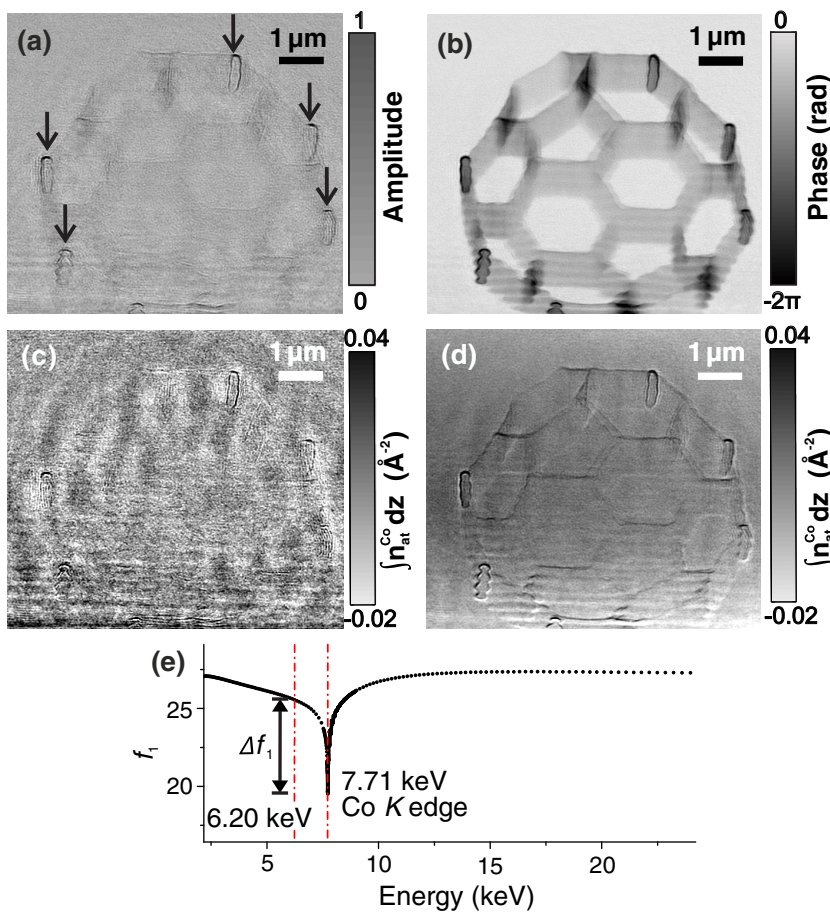

FIG. 2 (color online). Reconstructed (a) amplitude and (b) phase of the 2D complex-valued transmission function at an x-ray photon energy of $6.20 \mathrm{keV}$. The high-contrast cobalt layers on the bars parallel to the x-ray beam in the amplitude projection are indicated with arrows. The integrated atomic density of Co, $\int n_{\mathrm{at}}^{\mathrm{Co}} d z$, calculated from (c) the absorption and (d) the phase, respectively. (e) The real part of the Co scattering factor, $f_{1}$, encompassing the Co $K$ edge, with the change in $f_{1}$ between the two energies, $\Delta f_{1}$, indicated. 
where $r_{\mathrm{e}}$ is the classical electron radius, $n_{\mathrm{at}}^{k}$ is the atomic density of the $k$ th element, and we sum over all of the elements present. Far from any resonant energy, $\sum_{k} n_{\text {at }}^{k} f_{1}^{k}$ is the electron density. It is only near resonant energies that this term depends on the wavelength and is reduced due to anomalous scattering effects $[27,28]$. It is this anomalous scattering that allows the atomic density of a specific element to be extracted when taking the difference between on- and off-resonance measurements, as nonresonant elements do not contribute to this difference [27]. The atomic density can then be obtained from

$$
n_{\mathrm{at}}^{k}=\frac{1}{\Delta f_{1}^{k}} \frac{2 \pi}{r_{\mathrm{e}}}\left(\frac{\delta_{\text {off }}}{\lambda_{\text {off }}^{2}}-\frac{\delta_{\text {on }}}{\lambda_{\text {on }}^{2}}\right)
$$

with off and on denoting off resonance and on resonance, respectively, and where $\Delta f_{1}^{k}$ can be calculated from absorption data from bulk reference samples by making use of the Kramers-Kronig relation [27].

Using this method, we can determine the location of the cobalt in 2D by extracting its atomic density integrated along the x-ray direction $\int n_{\mathrm{at}}^{\mathrm{Co}} d z$. Thus far, elementspecific imaging involving resonant energies has primarily employed the absorption information of a sample to characterize various material properties. Here we demonstrate the effectiveness of the phase in resonant experiments. A single projection provides the real or imaginary part of $\int\left[\sum_{k} n_{\mathrm{at}}^{k}\left(f_{1}^{k}+j f_{2}^{k}\right)\right] d z$, calculated from the reconstructed phase or amplitude, respectively. By taking the difference between projections taken at two different energies, either for the phase or for the amplitude, with one energy tuned to the resonant element, Co, and dividing through by $\Delta f_{1}^{\mathrm{Co}}$ or $\Delta f_{2}^{\mathrm{Co}}$, we can obtain $\int n_{\mathrm{at}}^{\mathrm{Co}} d z$ calculated from the phase or amplitude, respectively. To obtain $\Delta f_{1}^{\mathrm{Co}}$, the real part of the atomic scattering factor $f_{1}^{\mathrm{Co}}$, shown in Fig. 2(e) with $\Delta f_{1}^{\text {Co }}$ indicated, was calculated using the Kramers-Kronig relation from $f_{2}^{\text {Co }}$ values consisting of theoretical absorption data supplemented by real absorption data taken around the $K$ edge energy [27]. After postprocessing of the ptychography reconstructions involving the removal of constant and linear phase terms [29], the pixel size of the $6.20 \mathrm{keV}$ projection, $21.48 \mathrm{~nm}$, was interpolated to that of $7.71 \mathrm{keV}, 17.27 \mathrm{~nm}$, and the projections were aligned to a small fraction of a pixel [30]. The difference of the two projections was then taken and normalized by $\Delta f_{1}^{\mathrm{Co}}$ and $\Delta f_{2}^{\mathrm{Co}}$. Projections of $\int n_{\mathrm{at}}^{\mathrm{Co}} d z$ showing the location of the Co in 2D calculated from the amplitude and the phase are given in Figs. 2(c) and 2(d), respectively, where the improved quality and lower noise of the density derived from the phase become evident.

In order to fully determine the $3 \mathrm{D}$ distribution of $n_{\mathrm{at}}^{\mathrm{Co}}$, we turn to the on- and off-resonance phase tomograms. The $3 \mathrm{D}$ distributions of the refractive index $\delta(\mathbf{r})$ of the sample at the two photon energies were obtained by postprocessing, alignment, and reconstruction of ptychographic projections following the procedure in Ref. [29], from which tomograms of $\sum_{k} n_{\mathrm{at}}^{k} f_{1}^{k}$ were calculated using Eq. (2). Horizontal slices through the tomograms are shown in Figs. 3(a) and 3(b), where both the polymer scaffold and the cobalt layer on the outside of the scaffold are clearly visible.

Analogous to the 2D case, the off-resonance tomogram was interpolated to the voxel size of the on-resonance tomogram, $17.27 \mathrm{~nm}$, using cubic interpolation and the $3 \mathrm{D}$ data sets aligned with subpixel resolution by a combination of 2D subpixel image registration [30] and alignment of vertical mass distribution functions similar to the method described in Ref. [29]. Using regions of air as a reference, we removed a small relative offset of $0.0044 \AA^{-3}$ in $\sum_{k} n_{\text {at }}^{k} f_{1}^{k}$. The 3D atomic density distribution of cobalt within the sample was computed by taking the difference between the two tomograms and dividing by $\Delta f_{1}=6.029$, as in Eq. (3), revealing the location of the cobalt-containing voxels, shown in Fig. 3(c).

In determining the spatial resolution, the Fourier shell correlation [31] gives a pessimistic estimate of $55 \mathrm{~nm}$ as the sample is mostly empty. Instead we obtain a more realistic value of $25 \mathrm{~nm}$ given by the full width at half maximum of the line profile taken across the thinnest cobalt layer in Fig 3(c), as shown in Fig. 3(d).

In addition to the Co distribution within the sample, knowledge of both the cobalt atomic density and electron density of the sample components gives added insight into the electronic state of the Co. Figure 4(a) shows a bivariate histogram of the electron density, $\sum_{k} n_{\mathrm{at}}^{k} f_{1}^{k}$ off resonance at $6.20 \mathrm{keV}$, and the cobalt atomic density, which reveals the relationship between the two densities for individual pixels.
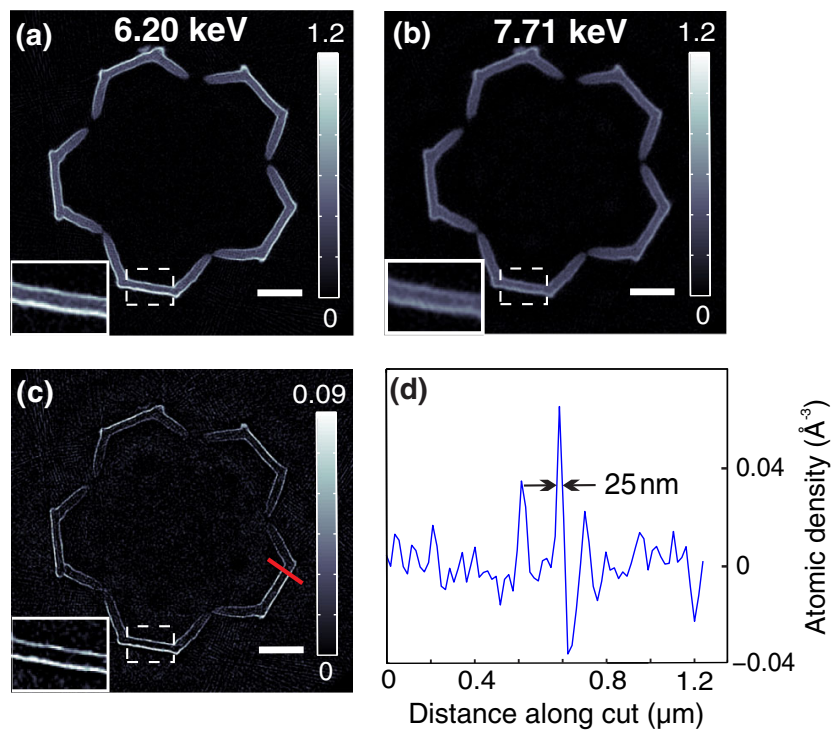

FIG. 3 (color online). Tomographic axial slice of $\sum_{k} n_{\text {at }}^{k} f_{1}^{k}$ in $\AA^{-3}$ (a) off resonance at $6.20 \mathrm{keV}$ and (b) on resonance at $7.71 \mathrm{keV}$. (c) Tomographic axial slice of the atomic density of Co, $n_{\mathrm{at}}^{\mathrm{Co}}$, within the sample. A line profile taken along the red line is shown in (d) with a FWHM of $25 \mathrm{~nm}$. Insets in (a)-(c) show a magnified portion of the slice. Scale bars represent $1 \mu \mathrm{m}$. 
The histogram shows a broad peak at the origin corresponding to air that can be clearly separated from the peak of the resist. The cobalt layer thickness is similar to the achieved spatial resolution and hence the tomogram suffers from considerable partial-volume effects, where voxels contain a combination of $\mathrm{Co}$ and resist or air. Therefore, instead of a well-defined peak in the histogram, the cobalt layer is present as a marked plume emerging from both the air and resist peaks. For further analysis, we segment the different material phases within this sample as indicated by the dashed white lines on the histogram in Fig. 4(a). The Co layer was segmented from the other materials using an $n_{\text {at }}$ value of $0.09 / \AA^{3}$ corresponding to that of bulk Co. The phases are rendered in Fig. 4(b) with the resist shown in blue and the Co in orange. The resist visible on the inside and underside of the buckyball illustrates the shadowing effects of the Co sputter deposition.

We look quantitatively at the electron density values in order to obtain more material-specific information about the sample. The mean electron density within the resist phase was measured to be $(0.46 \pm 0.16) / \AA^{3}$ at $6.20 \mathrm{keV}$, consistent with the expected electron density of a
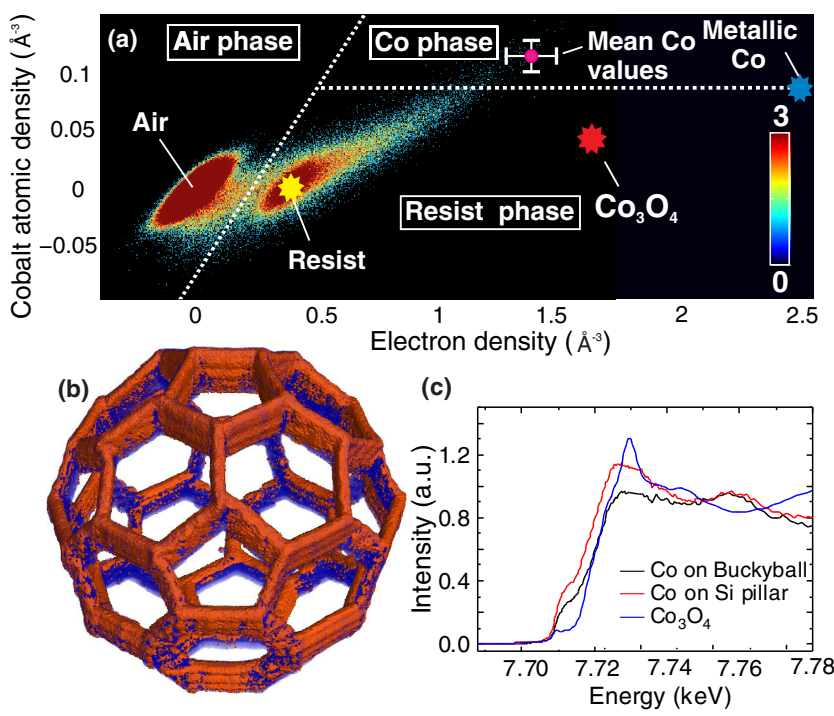

FIG. 4 (color online). (a) A bivariate histogram showing the number of pixels as a function of the electron, $n_{\mathrm{e}}$, and cobalt atomic, $n_{\mathrm{at}}^{\mathrm{Co}}$, densities using a logarithmic scale. The segmentation of air, resist, and cobalt is indicated by the dashed white lines with the phases labeled accordingly. Peaks corresponding to air and resist are labeled, and the blue, red, and yellow stars indicate the expected values for bulk $\mathrm{Co}, \mathrm{Co}_{3} \mathrm{O}_{4}$, and resist, respectively, while the mean value of $n_{\mathrm{at}}^{\mathrm{Co}}$ and $n_{\mathrm{e}}^{\mathrm{Co}}$ measured in Co-containing voxels is shown. The mean has error bars of $0.02 / \AA^{3}$ and $0.11 / \AA^{3}$ for $n_{\mathrm{at}}^{\mathrm{Co}}$ and $n_{\mathrm{e}}^{\mathrm{Co}}$, respectively, representing the standard deviation of the measured values. (b) The $3 \mathrm{D}$ rendering of the tomogram with elemental contrast, where the $\mathrm{Co}$ is rendered in orange and the resist in blue. (c) The fluorescence spectra for the cobalt on the buckyball and, as a reference, for a point on the Co-coated $\mathrm{Si}$ pillar. A spectrum for $\mathrm{Co}_{3} \mathrm{O}_{4}$, measured in transmission, is also given for comparison. cross-linked polymer. To reduce partial volume effects, we calculate the electron density of the Co from Co-containing voxels surrounded only by Co. The mean value of the electron density within these areas was found to be $(1.39 \pm 0.11) / \AA^{3}$, indicated in Fig. 4(a), with a maximum of $(1.64 \pm 0.17) / \AA^{3}$, much more similar to the electron density of $\mathrm{Co}_{3} \mathrm{O}_{4}, 1.66 / \AA^{3}$, than that of metallic Co, $2.44 / \AA^{3}$, giving an indication of the sample being oxidized.

For the Co, a higher mean value than expected for the atomic density of $(0.119 \pm 0.020) / \AA^{3}$ was measured. This systematic error in $n_{\mathrm{at}}^{\mathrm{Co}}$ could be due to radiation damage to the polymer scaffold introducing structural disorder as well as diluting or further oxidizing the Co over time. As the on-resonance measurements were performed after the off-resonance measurements, the lowering of the electron density with time would result in a higher calculated value of the atomic density, consistent with our experimental results. The presence of radiation damage is supported by measurements of the electron density of the polymer phase, $n_{\mathrm{e}}^{\text {poly }}$, at the two energies, where a ratio of 0.92 of $n_{\mathrm{e}}^{\text {poly }}(7.71 \mathrm{keV}) /$ $n_{\mathrm{e}}^{\text {poly }}(6.20 \mathrm{keV})$ is found, indicating that there is indeed a modification of the polymer in the $\mathrm{X}$-ray beam.

The oxidation state of the Co was confirmed with microfluorescence spectroscopy experiments performed at the PHOENIX beam line, Swiss Light Source, Paul Scherrer Institute, Switzerland. A focused beam of 4-5 $\mu \mathrm{m}$ in size was used to measure the fluorescence spectrum across the $K$ edge of $\mathrm{Co}$ at several points across the sample, both on the buckyball and, as a reference, on the Co-coated Si pillar. Comparing spectra taken for Co on the buckyball and on the Si pillar, shown in Fig. 4(c), a blue shift of the edge and a broadening of the white line are observed, indicating the presence of oxidation and disorder in the Co film on the buckyball, respectively, in agreement with our resonant ptychographic tomography results.

To conclude, in this Letter we have presented full structural and elemental 3D characterization at the nanoscale, achieved by performing resonant ptychographic tomography and exploiting resonant anomalous elastic scattering. We determined the location of Co atoms in a $6 \mu \mathrm{m}$ artificial buckyball to within a spatial resolution of $25 \mathrm{~nm}$, and quantitative data revealed that the Co layer on our test sample was predominantly oxidized. Resonant ptychographic tomography enables element-specific characterization, which can be extended to many elements within a sample by taking additional measurements at their corresponding absorption edges. This combination of tomography with resonant scattering opens the door to the full determination of 3D electronic and magnetic functionality of materials, spatially resolved at the nanoscale.

The authors thank Anja Weber, Robert Kirchner, and Victor Cadarso for their help with sample preparation and Ana Diaz, Julio C. da Silva (supported by SNF Grant No. 137772), as well as Christian David, Olga Safonova 
and Riccardo Hertel for fruitful discussions. X-ray optical elements used at the cSAXS beam line were fabricated at the Laboratory for Micro- and Nanotechnology, Paul Scherrer Institute, Switzerland. The instrumentation was supported by SNF (R'EQUIP, 145056, OMNY) and the Competence Centre for Materials Science and Technology (CCMX) of the ETH-Board, Switzerland. Part of this work was performed at the cSAXS and PHOENIX beam lines of the Swiss Light Source, Paul Scherrer Institute, Switzerland.

*manuel.guizar-sicairos@psi.ch

†laura.heyderman@psi.ch

[1] G. von Freymann, A. Ledermann, M. Thiel, I. Staude, S. Essig, K. Busch, and M. Wegener, Three-Dimensional Nanostructures for Photonics, Adv. Funct. Mater. 20, 1038 (2010).

[2] B. J. Nelson, I. K. Kaliakatsos, and J. J. Abbott, Microrobots for Minimally Invasive Medicine, Annu. Rev. Biomed. Eng. 12, 55 (2010).

[3] J. K. Gansel, M. Thiel, M. S. Rill, M. Decker, K. Bade, V. Saile, G. von Freymann, S. Linden, and M. Wegener, Gold Helix Photonic Metamaterial as Broadband Circular Polarizer, Science 325, 1513 (2009).

[4] A. Blanco et al., Large-scale synthesis of a silicon photonic crystal with a complete three-dimensional bandgap near 1.5 micrometres, Nature (London) 405, 437 (2000).

[5] S. S. P. Parkin, M. Hayashi, and L. Thomas, Magnetic Domain-Wall Racetrack Memory, Science 320, 190 (2008).

[6] R. Hertel, Curvature-Induced Magnetochirality, SPIN 03, 1340009 (2013).

[7] T. Bueckmann, N. Stenger, M. Kadic, J. Kaschke, A. Frölich, T. Kennerknecht, C. Eberl, M. Thiel, and M. Wegener, Tailored 3D Mechanical Metamaterials Made by Dip-in Direct-Laser-Writing Optical Lithography, Adv. Mater. 24, 2710 (2012).

[8] S. Tottori, L. Zhang, F. Qiu, K. K. Krawczyk, A. FrancoObregón, and B.J. Nelson, Magnetic Helical Micromachines: Fabrication, Controlled Swimming, and Cargo Transport, Adv. Mater. 24, 811 (2012).

[9] E. Gallucci, K. Scrivener, A. Groso, M. Stampanoni, and G. Margaritondo, 3D experimental investigation of the microstructure of cement pastes using synchrotron X-ray microtomography $(\mu \mathrm{CT})$, Cement and Concrete Research 37, 360 (2007).

[10] M. Beckers, T. Senkbeil, T. Gorniak, M. Reese, K. Giewekemeyer, S.-C. Gleber, T. Salditt, and A. Rosenhahn, Chemical Contrast in Soft X-Ray Ptychography, Phys. Rev. Lett. 107, 208101 (2011).

[11] A. M. Maiden, G. R. Morrison, B. Kaulich, A. Gianoncelli, and J. M. Rodenburg, Soft X-ray spectromicroscopy using ptychography with randomly phased illumination, Nat. Commun. 4, 1669 (2013).

[12] R. Hoppe, J. Reinhardt, G. Hofmann, J. Patommel, J.-D. Grunwaldt, C. D. Damsgaard, G. Wellenreuther, G. Falkenberg, and C. G. Schroer, High-resolution chemical imaging of gold nanoparticles using hard x-ray ptychography, Appl. Phys. Lett. 102, 203104 (2013).
[13] A. Diaz, P. Trtik, M. Guizar-Sicairos, A. Menzel, P. Thibault, and O. Bunk, Quantitative X-ray phase nanotomography, Phys. Rev. B 85, 020104 (2012).

[14] J. M. Rodenburg, A. Hurst, A. Cullis, B. Dobson, F. Pfeiffer, O. Bunk, C. David, K. Jefimovs, and I. Johnson, Hard-XRay Lensless Imaging of Extended Objects, Phys. Rev. Lett. 98, 034801 (2007).

[15] P. Thibault, M. Dierolf, A. Menzel, O. Bunk, C. David, and F. Pfeiffer, High-Resolution Scanning X-ray Diffraction Microscopy, Science 321, 379 (2008).

[16] M. Guizar-Sicairos and J. R. Fienup, Phase retrieval with transverse translation diversity: a nonlinear optimization approach, Opt. Express 16, 7264 (2008).

[17] A. M. Maiden and J. M. Rodenburg, An improved ptychographical phase retrieval algorithm for diffractive imaging, Ultramicroscopy 109, 1256 (2009).

[18] J. M. Rodenburg, Ptychography and Related Diffractive Imaging Methods, Adv. Imaging Electron Phys. 150, 87 (2008).

[19] D. A. Shapiro et al., Chemical composition mapping with nanometre resolution by soft X-ray microscopy, Nat. Photonics 8, 765 (2014).

[20] M. Holler, A. Diaz, M. Guizar-Sicairos, P. Karvinen, E. Färm, E. Härkönen, M. Ritala, A. Menzel, J. Raabe, and O. Bunk, X-ray ptychographic computed tomography at $16 \mathrm{~nm}$ isotropic 3D resolution, Sci. Rep. 4, 3857 (2014).

[21] F. Laermer and A. Schilp, U.S. Patent No. 5501893 (1996).

[22] M. Dierolf, A. Menzel, P. Thibault, P. Schneider, C. M. Kewish, R. Wepf, O. Bunk, and F. Pfeiffer, Ptychographic X-ray computed tomography at the nanoscale, Nature (London) 467, 436 (2010).

[23] M. Holler, J. Raabe, A. Diaz, M. Guizar-Sicairos, C. Quitmann, A. Menzel, and O. Bunk, An instrument for 3D x-ray nano-imaging, Rev. Sci. Instrum. 83, 073703 (2012).

[24] B. Henrich, A. Bergamaschi, C. Broennimann, R. Dinapoli, E. F. Eikenberry, I. Johnson, M. Kobas, P. Kraft, A. Mozzanica, and B. Schmitt, PILATUS: A single photon counting pixel detector for X-ray applications, Nucl. Instrum. Methods Phys. Res., Sect. A 607, 247 (2009).

[25] P. Kraft et al., Characterization and Calibration of PILATUS Detectors, IEEE Trans. Nucl. Sci. 56, 758 (2009).

[26] P. Thibault and M. Guizar-Sicairos, Maximum-likelihood refinement for coherent diffractive imaging, New J. Phys. 14, 063004 (2012).

[27] F. Hippert et al., Neutron and X-ray Spectroscopy (Springer, New York, 2006), Chap. 7.

[28] R. W. James, The Optical Principles of the Diffraction of X-rays (G. Bell and Sons Ltd., London, 1962), Chaps. 3 and 4.

[29] M. Guizar-Sicairos, A. Diaz, M. Holler, M. S. Lucas, A. Menzel, R. A. Wepf, and O. Bunk, Phase tomography from $\mathrm{X}$-ray coherent diffractive imaging projections, Opt. Express 19, 21345 (2011).

[30] M. Guizar-Sicairos, S. T. Thurman, and J. R. Fienup, Efficient subpixel image registration algorithms, Opt. Lett. 33, 156 (2008).

[31] M. van Heel and M. Schatz, Fourier shell correlation threshold criteria, J. Struct. Biol. 151, 250 (2005). 